\title{
In Situ TEM Observation of AlGaN/GaN HEMTs Under Applied DC Bias
}

\author{
A.C. Lang, C.R. Winkler, J.D. Sloppy, C.L. Johnson, and M.L. Taheri \\ Department of Materials Science \& Engineering, Drexel University, Philadelphia, PA 19104
}

Unlike conventional semiconductors, high electron mobility transistors (HEMTs) are field effect transistors that take advantage of the electron layer at the interface between two different semiconductor materials (with different band gaps). GaN HEMTs are the backbones of many high frequency applications [1-8]. There are multiple types of HEMTs, of which, AlGaAs/GaAs types are the most common. However, $\mathrm{AlGaN} / \mathrm{GaN}$ types are of interest due to the wide band gap of $\mathrm{GaN}$ and the ability of $\mathrm{AlGaN} / \mathrm{GaN}$ to carry high voltage and current. The resulting heterostructure exhibits superior carrier saturation velocity, thermal conductivity, and high breakdown field, all of which are required for high temperature and high speed applications [1-8]. AlGaN/GaN HEMTs are significantly different from other HEMT structures due to a very strong piezoelectric effect caused by the lattice mismatch between $\mathrm{AlGaN}$ and GaN. This lattice mismatch, in addition to spontaneous polarization fields, give rise to an interfacial charge that is referred to as a two-dimensional electron gas (2DEG) [1-8]. In order to increase reliability and performance of AlGaN/GaN structures, the electrical degradation of these HEMTs need to be examined under an electric field bias. By utilizing in situ TEM, we plan to clarify the degradation mechanisms associated with defects at the 2DEG by direct observation of their nucleation under applied DC bias.

Experiments will be conducted in order to investigate the drain interface region, piezoelectricinduced strain, and relaxation trap region in the AlGaN layer beneath the gate region. A diagram of the experimental design for the in situ TEM studies is shown in figure 1. Samples will be tested below, at, and above critical voltage values, which have been experimentally determined to be approximately 40V [1-8]. The experiments will allow for the monitoring of the evolution of defects associated with GaN HEMT electrical degradation. Specifically, the data generated will give rise to predictive mechanistic models of the evolution of defects under various electrical stress conditions. This will contribute to improved reliability of HEMT devices. 


\section{References}

[1] Y. Zhang et al., J. Appl. Phys. 85 (1), (1999) 587.

[2] DARPA Report, "WBGS-RF GaN-on-SiC Program: The Next Generation of MMICs", Mark Rosker, 2009.

[3] Hsu et al., Appl. Phys. Lett., 81( 1). (2002) 79.

[4] Makaram et al., Appl. Phys. Lett. 96. (2010) 233509.

[5] J.A. del Alamo, Microelectronics Reliability 49. (2009) 1200.

[6] S.Y. Park et al., Microelectronics Reliability 49. (2009) 478.

[7] U. Chowdhury et al., IEEE Elec. Dev. Lett., 29(10). (2008) 1098.

[8] G. Meneghesso et al., IEEE Transactions on Device and Materials Reliability, 8(2). (2008) 332.

[9] This research was supported by the Office of Naval Research through code 312"Electronics, Sensors, and Network Research," under contract number N000141110296 (Program Manager Daniel Green). David Meyer and Steve Binari of the Naval Research Laboratory in Washington, DC, are gratefully acknowledged for providing HEMT devices.

\section{Electron Beam Perpendicular to Sample}

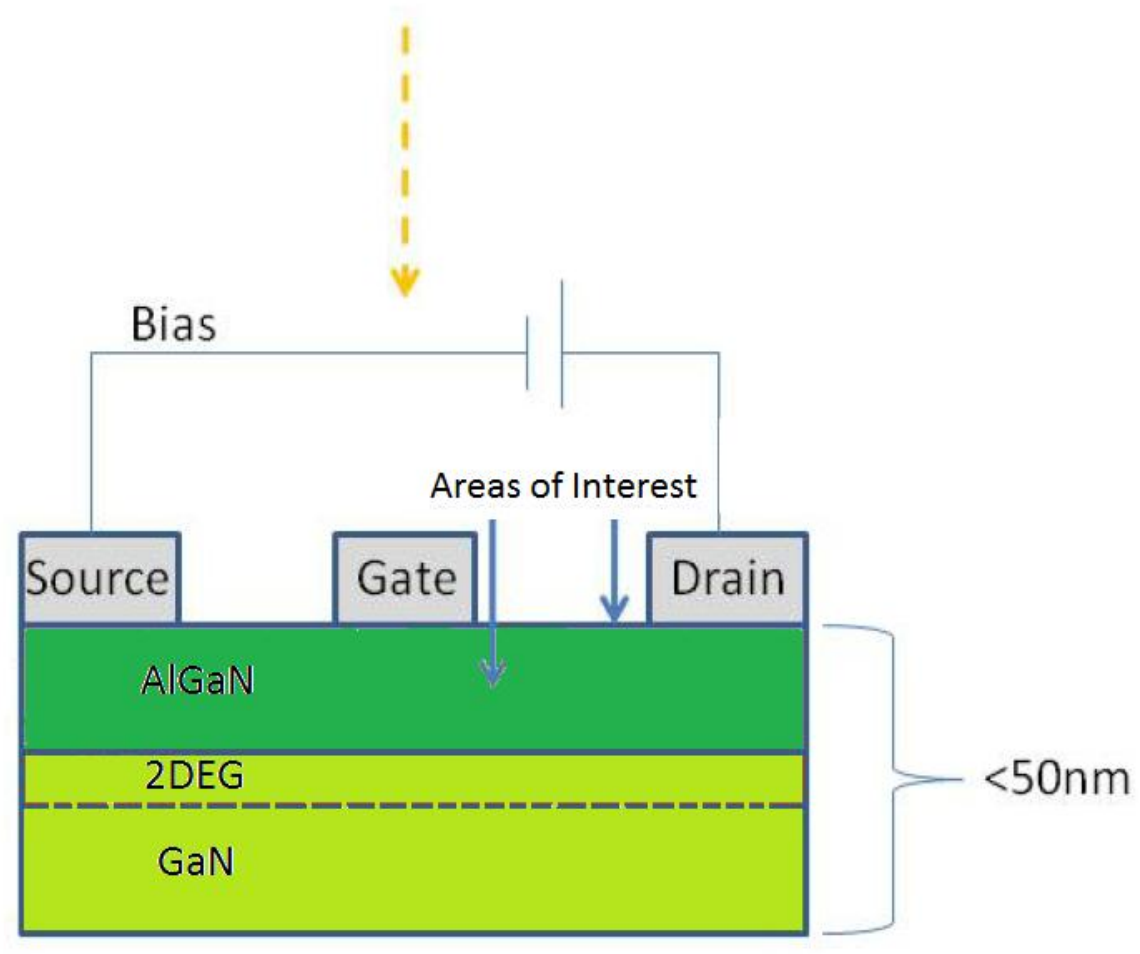

Figure 1: Experimental design for in situ TEM degradation studies of $\mathrm{AlGaN} / \mathrm{GaN}$ HEMT device heterostructures. 